\title{
Next Generation Sequencing: The Current Challenge is the Translation into Clinics
}

\author{
Jesus Mates ${ }^{1}$, Bernat Del Olmo¹, Carles Ferrer-Costa ${ }^{2}$, Catarina Allegue1, Ramon Brugada ${ }^{1,3,4}$ and Oscar Campuzano ${ }^{1,3 *}$ \\ ${ }^{1}$ Cardiovascular Genetics Center, University of Girona-IDIBGI, Girona, Spain \\ ${ }^{2}$ Gendiag SL, Barcelona, Spain \\ ${ }^{3}$ Department of Medical Sciences, School of Medicine, University of Girona, Girona, Spain \\ ${ }^{4}$ Cardiovascular Genetics Unit, Hospital Josep Trueta, Girona, Spain
}

\section{Editorial}

It is a fact that we are immersed in the Next Generation Sequencing (NGS) era. At this stage that does not catch anyone off guard. The publication of the human genome sequence in 2001 was the start of an unprecedented scientific production, only comparable to that produced on 1953, with the discovery of three-dimensional structure of the DNA double helix

The improvement in the high throughput sequencing techniques in last years, the emergence of a wide range of affordable sequencing platforms and the reagent cost reduction have allowed to perform a cost-effectives genetic analysis in a reduced time [1]. Hence, regarding PubMed website, of the approximately 18000 topic-related papers published in last 15 years, nearly 17000 has been published in last 5 years. The available variety and widespread use of modern sequencing techniques make these publications capable of bringing something new to an open and plastic scientific community which has to assimilate scientific breakthroughs in increasingly shorter periods of time [2].

Biomedical arena is progressively including gene panels in research as well as diagnostics. This approach is useful to identify common and rare genetic alterations in a subset of selected genes, and it is particularly best suited for known Mendelian diseases that can be phenotypically explained with the pathogenic variant found in the regions of interest [3]. Unfortunately, rare and complex diseases cannot be fully diagnosed using this approach due to the limited size, and often many cases remain unconcluded after being analyzed. In these cases, a wider analysis provided by Whole-Exome Sequencing (WES) or Whole-Genome Sequencing (WGS) can reveal the cause.

However, current guidelines/recommendations of most part of diseases do not include NGS as part of the diagnosis, as occurs in inherited cardiac genetic diseases, for example [4]. Hence, more than 100 genes associated with sudden cardiac death have been described, but only 20 are currently recommended. The main reason for this fact is the interpretation of the large amount of data generated by NGS technologies, and translation into clinical practice [5].

Are the current genetic diagnosis guidelines the most appropriate? We know that there are a significant percentage of clinical cases which can be perfectly explained by the screening of certain genes and the identification of specific variants. But there is an elevated percentage of cases for a broad spectrum of diseases that remain unexplained.

The current challenge is the correct interpretation of Variants of Uncertain/Unknown Significance (VUS). The only way to clarify their role is complementing the NGS screening data with RNA-seq and Chip-seq experiments. In that way we are going to be able to detect the real effect of the hypothetic pathogenic variant on its own affected tissue. Moreover, the modern CRISPR/Cas9 [6,7] technique for genome editing altogether with more realistic functional models like Induced Pluripotent Stem (IPS) cells [8] may help to elucidate the real role of this kind of variants.
Moving towards this reality the scientific community has embarked on some ambitious projects of population sequencing such as the 1000 Genomes Project [9], the UK10K [10] or the ENCODE project [11]. All this scientific efforts are directed to achieve a better understanding of the human genome, it's regulation and the relation between the low frequency variants and the pathogenic ones. So far all this work has been relegated to research purposes. Perhaps it is still early to implement all this data into the clinical genetic diagnose and counseling but by the time it will be essential.

\section{References}

1. van Dijk EL, Auger H, Jaszczyszyn $Y$, Thermes $C$ (2014) Ten years of nextgeneration sequencing technology. Trends Genet 30: 418-426.

2. Xuan J, Yu Y, Qing T, Guo L, Shi L (2013) Next-generation sequencing in the clinic: promises and challenges. Cancer Lett 340: 284-295.

3. Sikkema-Raddatz B, Johansson LF, de Boer EN, Almomani R, Boven LG, et al (2013) Targeted next-generation sequencing can replace Sanger sequencing in clinical diagnostics. Hum Mutat 34: 1035-1042.

4. Priori SG, Wilde AA, Horie M, Cho Y, Behr ER, et al. (2013) HRS/EHRA APHRS expert consensus statement on the diagnosis and management of patients with inherited primary arrhythmia syndromes: document endorsed by HRS, EHRA, and APHRS in May 2013 and by ACCF, AHA, PACES, and AEPC in June 2013. Heart Rhythm 10: 1932-1963.

5. Allegue C, Coll M, Mates J, Campuzano O, Iglesias A, et al. (2015) Genetic Analysis of Arrhythmogenic Diseases in the Era of NGS: The Complexity of Clinical Decision-Making in Brugada Syndrome. PLoS One 10: e0133037.

6. Jinek M, Chylinski K, Fonara I, Hauer M, Doudna JA (2012) A programmable dual-RNA-guided DNA endonuclease in adaptive bacterial immunity. Science 337: 816-821.

7. Cong L, Ran FA, Cox D, Lin S, Barretto R, et al. (2013) Multiplex genome engineering using CRISPR/Cas systems. Science 339: 819-823.

8. Dell'Era P, Benzoni P, Crescini E, Valle M, Xia E, et al. (2015) Cardiac disease modeling using induced pluripotent stem cell-derived human cardiomyocytes. World J Stem Cells 7: 329-342.

9. Auton A, Brooks LD, Durbin RM, Garrison EP, Kang HM, et al. (2015) A global reference for human genetic variation. Nature 526: 68-77.

10. http://www.uk10k.org

11. https://www.encodeproject.org

*Corresponding author: Oscar Campuzano, Cardiovascular Genetics Center University of Girona-IDIBGI, Girona, Spain, Tel: +34 8729870 87, Extn. 63; E-mail: oscar@brugada.org

Received September 28, 2015; Accepted October 01, 2015; Published October 05, 2015

Citation: Campuzano O, Mates J, Olmo BD, Costa CF, Allegue C, et al. (2015) Next Generation Sequencing: The Current Challenge is the Translation into Clinics. Next Generat Sequenc \& Applic 2: e105. doi:10.4172/2469-9853.1000e105

Copyright: (๑) 2015 Campuzano O, et al. This is an open-access article distributed under the terms of the Creative Commons Attribution License, which permits unrestricted use, distribution, and reproduction in any medium, provided the original author and source are credited. 